\title{
Frequency of the hemochromatosis gene $(H F E)$ variants in a Jordanian Arab population and in diabetics from the same region
}

\author{
Asem Alkhateeb, Amal Uzrail and Khaldon Bodoor \\ Biotechnology and Genetic Engineering Department, Jordan University of Science and Technology, P.O.Box 3030, \\ Irbid 22110, Jordan \\ E-mail: asemalkhateeb@just.edu.jo
}

\begin{abstract}
Hereditary $H F E$-linked hemochromatosis is a frequent recessive disorder among individuals of northern European ancestry. The clinical characteristic of this disease is the gradual accumulation of iron in internal organs, which ultimately may lead to organ damage and death. Three allelic variants of $H F E$ gene have been correlated with hereditary hemochromatosis: C282Y is significantly associated with hereditary hemochromatosis in populations of Celtic origin, H63D and S65C are associated with milder form of iron overload. In this study we performed mutation analysis to identify allele frequency of the three variants of $H F E$ gene in Jordanian Arab population, to assess deviations of these frequencies from those detected elsewhere, and to determine if there is an increased frequency of these variants in a diabetic population (Type 2 diabetes) from the same area. DNA was extracted from blood samples of 440 individuals attending King Abdullah University Hospital for ambulatory services. We used polymerase chain reaction (PCR) to amplify exons 2 and 4 of the $H F E$ gene then restriction fragment length polymorphism (RFLP) method to detect the variants. There were neither homozygous nor heterozygous for C282Y variant. For the H63D variant, $0.68 \%$ were homozygous and $21.1 \%$ were heterozygous. For the S65C variant, there were no homozygous and $0.23 \%$ were heterozygous. Allelic frequencies were, $0 \%, 11.25 \%$, and $0.11 \%$ for C282Y, H63D, and S65C, respectively. Our samples were subdivided into two categories of type 2 diabetic ( 89 cases) and controls (blood donors, 204 cases) and compared with regard to the H63D variant. Both groups did not have homozygous H63D variant. H63D heterozygous in diabetics were $23.60 \%$ and in blood donor controls $22.55 \%$. Allelic frequency of the mutant H63D allele was $11.80 \%$ in diabetics and $11.27 \%$ for the blood donor controls. This is the first study to show the frequency of the three hemochromatosis gene variants in Jordan with the interesting finding of no $\mathrm{C} 282 \mathrm{Y}$ allele detected in 440 samples. Additionally, no significant difference was observed in H63D variant frequency in type 2 diabetics as compared to controls.
\end{abstract}

Keywords: Hemochromatosis, HFE, diabetes, H63D

\section{Introduction}

Hereditary HFE-linked hemochromatosis (OMIM 235200) is an autosomal recessive disorder of iron metabolism. It is caused by an increased absorption of iron from the diet and consequent deposition of the extra iron in parenchymal cells of the liver, pancreas and heart. This deposition leads to abnormality in organ functions and results in multiple manifesta- tions including primarily; liver cirrhosis, diabetes mellitus, hyperpigmentation of skin, and cardiac failure (reviewed in 1). Phenotypic expression usually starts with fatigue followed by symptomatic organ involvement of liver, heart, endocrine system and joint disease. Hemochromatosis gene, HFE, was discovered in 1996, with homozygosity for C282Y (c.845G > A, rs1800562) variant being most commonly associated with the disease [2]. C282Y seem to have originat- 
ed in north Europe around 2000 years ago and spread since then [3]. The frequency of this variant ranges from $14 \%$ in Ireland [4] to $0 \%$ in different countries [5]. Two other variants in $H F E$ are thought to increase the risk of hemochromatosis namely; H63D (c.187C > G, rs1799945) and S65C (c.193A > T, rs1800730). Hemochromatosis has a low penetrance [6]. It has an age of onset in midlife, being later in females than males.

Diabetes mellitus is the most commonly associated endocrine abnormality with hemochromatosis. Not all hemochromatosis patients develop diabetes mellitus but the high frequency of association prompted us to explore whether mutations in $H F E$ could increase the risk of developing diabetes mellitus in different populations.

The frequency of hemochromatosis gene variants was never studied in the Jordanian Arab population. Jordan contains a homogenous Arab population but received multiple migrations and population admixture. In this study we carried a population frequency analysis for HFE gene variants, C282Y, H63D, and S65C in 440 Jordanian Arabs. We also investigated the association of H63D variant with diabetes to determine if there is an increased frequency in diabetic patients from the same population.

\section{Subjects and methods}

\subsection{Subjects}

A total of 440 individuals have participated in this study. Subjects were recruited from individuals attending a major hospital that serves the north part of Jordan (King Abdullah University Hospital, Irbid). The largest group of subjects were blood donors with no abnormality as required by blood donors department (No diabetes, blood disorders, cardiovascular disorders, etc.). The other groups were patients attending the hospital, none of which had a hemochromatosis diagnosis. Classification of cases: 204 blood donors (age range: $18-49$, average: 26.7 years), 89 type 2 diabetes (age range: 15-73, average: 54.7 years), 42 patients undergoing hemodialysis (age range: $11-81$, average: 49.3 years), 29 thalassemia patients (age range: $2-30$, average: 16.3 years), 29 patients attending the gynecology and fertility clinic (age range: 18-48, average: 33.4 years), 11 arthritis patients (age range: $23-59$, average: 44.0 years) and 36 with miscellaneous indications including endocrine problems and autoimmunity.
All 440 subject samples were used in the allele frequency analysis. Type 2 diabetes samples were used for the association analysis with blood donor samples as controls. Information regarding age, sex, and status of care needed were recorded. The mean age of population studied was 35.4 years, range 2-90 years. Positive control samples with homozygous mutants and heterozygous mutants were kindly provided by Dr. Osama Smadi (King Faisal hospital, Saudi Arabia) and Dr. Richard Allen (University of Oklahoma Health Sciences Center, USA). An informed consent was obtained from all participants or their legal guardians for children. This study was approved by the ethics committee (Institutional Review Board) of the Jordan University of Science and Technology.

\subsection{Genotyping}

Peripheral blood samples were collected from all participants. DNA was extracted using DNA purification Kits (Qiagen). Manufacturer's protocol was followed. Two amplicons were amplified by PCR for genotyping the three variants [7]. Primers were designed using primer3 software [8]. Sequence for primers used to genotype $\mathrm{C} 282 \mathrm{Y}$ were: forward 5'AAGGATAAGCAGCCAATGGA-3' and reverse 5'CCATAATTACCTCCTCAGGCACT-3' (GenBank Accession number NT_007592.15). To genotype H63D and S65C, same primer set was used: forward 5'GTTTGAAGCTTTGGGCTACG-3' and reverse 5'TACCCTTGCTGTGGTTGTGA-3' (GenBank Accession numberNT_007592.15). Amplifications were performed in a total volume of $25 \mu \mathrm{l}$ using the thermal cycler (icycler, Bio-Rad). Each PCR reaction contained $12.5 \mu \mathrm{l}$ GoTaq ${ }^{\circledR}$ Green Master mix, 2X (dNTPs, $\mathrm{MgCl} 2$, PCR buffer and Taq polymerase) (Promega), $2.5 \mu \mathrm{l}$ of each primer ( $1 \mu \mathrm{M}$ final concentration) (Alpha DNA), $4.5 \mu$ l nuclease free water, and $3.0 \mu \mathrm{l}$ (75 ng) of DNA template. After an initial step of 5 minutes at $95^{\circ} \mathrm{C}$, the samples were processed through 35 temperature cycles of 30 seconds at $94^{\circ} \mathrm{C}, 30$ seconds at $56^{\circ} \mathrm{C}$, and 30 seconds at $72^{\circ} \mathrm{C}$, and a final extension step of $72^{\circ} \mathrm{C}$ for 10 minutes. PCR products were run on $2 \%$ agarose gel and visualized by UV light after ethidium bormide staining to assess the correct sizing of the amplified product. Following PCR, products were digested by RsaI for C282Y, MboI for H63D and HinfI for S65C (Fermentas). The restriction fragments of PCR products were separated by electrophoresis on a $3 \%$ agarose gel containing $10 \mu \mathrm{g} / \mathrm{ml}$ ethidium bormide, and visualized by UV light and genotypes recorded. Positive and negative controls were included in each run. 
Table 1

Mutant genotypes HFE (C282Y, H63D and S65C) and their frequency in the Jordanian population; WT denotes wild type

\begin{tabular}{lcc}
\hline Genotype & Individuals with genotype & $\%$ \\
\hline C282Y/WT & 0 & 0 \\
C282Y/C282Y & 0 & 0 \\
H63D/WT & 93 & 21.1 \\
H63D/H63D & 3 & 0.68 \\
S65C/WT & 1 & 0.23 \\
S65C/S65C & 0 & 0 \\
Compound heterozygous & 0 & 0 \\
WT/WT & 343 & 78.0 \\
Total & 440 & 100 \\
\hline
\end{tabular}

\subsection{Statistical analysis}

Data were entered in Excel (Microsoft Corporation) for the calculation of allele and genotype frequencies. Hardy-Weinberg equilibrium (HWE) was tested to determine if the population was fulfilling the HWE at each variant locus. It was assessed in the observed genotype distribution with a Chi squared test. Allelic association $p$ values were determined using a Chi squared test between cases and controls. Genotypic association $p$ values were determined by the Freeman-Halton extension of Fisher's exact test for a $2 \times 3$ contingency table which evaluates the occurrence of all three genotypes as an array between the cases and controls [9]. A web-based calculator was used to compute $p$ values (Vassar Stats). A $p$ value $<0.05$ was considered to be statistically significant for both tests.

\section{Results}

Subjects participating in the study were 440, 202 (45.9\%) females and $238(54.1 \%)$ males. Blood donors constituted the major group $(204,46.4 \%)$, the other subjects had different indications; diabetes mellitus (89, $20.2 \%$ ), patients undergoing hemodialysis $(42,9.5 \%)$, thalassemia $(29,6.6 \%)$ patients attending the gynecology and fertility clinic $(29,6.6 \%)$, rheumatoid arthritis $(11,2.5 \%)$, and miscellaneous indications $(36,8.2 \%)$. Genotypes for the three $H F E$ variants were analyzed in all subjects representing the north part of the Jordanian population. The frequencies of genotypes of C282Y, H63D, and S65C are shown in Table 1. C282Y was not found in all 440 individuals genotyped. S65C was found only in one case with an allele frequency of $0.11 \%$. H63D was found to be a common allele in the Jordanian population with 93 heterozygotes $(21.1 \%)$, three homozygote mutants $(0.68 \%)$ and an allele frequency of $11.25 \%$. No compound heterozygotes were found in our population. All genotypes were consistent with Hardy-Weinberg expectations.

Data were further divided into two groups of type 2 diabetic ( 89 subjects, $62 \%$ females) and blood donors (204 subjects, 28\% females). Since only H63D has a considerable presence in the Jordanian population it was used to compare its frequency in type 2 diabetic population with blood donor population. The Blood donors represented the control population. H63D allele frequency of the blood donors (204 subjects) was $11.27 \%$, which is comparable to H63D frequency $(11.25 \%)$ in all 440 subjects. Genotype and allelic frequencies of the diabetic and blood donors control population are shown in table 3. No significant difference was found between H63D genotypes of the two groups $(p=0.84)$. Frequency of H63D heterozygous mutation in diabetic patients was $23.60 \%$ and in the control population $22.55 \%$. No H63D homozygote mutant was present in either population. Allele frequency of the mutant H63D allele was $11.80 \%$ in the diabetic population and $11.27 \%$ in the control population with no significant difference $(p=0.65)$.

\section{Discussion}

This is the first study to analyze hemochromatosis gene variants, C282Y, H63D, and S65C in Jordanian Arab population. $\mathrm{C} 282 \mathrm{Y}$ was not detected in all 440 samples tested. Frequency of H63D homozygous and heterozygous mutation was $0.68 \%$ and $22.1 \%$, respectively. S65C was detected with $0.23 \%$ heterozygous and no homozygous. Furthermore, H63D variant was tested for association with type 2 diabetes patients. No significant association of H63D allele was found in our diabetes patients as compared to matched controls.

Jordan is a small country in the Middle East with a relatively homogenous population. It was part of the Roman Empire until the 7th century and received much influx of crusader armies in the 11th and 12th centuries. Thus, admixture of Europeans with the indigenous population might be expected to spread the $\mathrm{C} 282 \mathrm{Y}$ variant that is thought to be generated north of Europe some 2000 years ago [10]. Unexpectedly, no C282Y allele was found in 440 individuals studied. This explains the rarity of hemochromatosis in Jordan. Total absence of $\mathrm{C} 282 \mathrm{Y}$ in Jordanians could indicate that the genetic mixture between European crusaders and the Arab population was non-existent or negligible and that currently there is negligible genetic mixture with northern Europeans. Another explanation could be that the ma- 
Table 2

Frequency of $H F E$ variants in different populations as compared to the Jordanian population

\begin{tabular}{lcccc}
\hline Population & \multicolumn{3}{c}{ Allele frequencies \% } & Reference \\
\cline { 2 - 4 } & C282Y & H63D & S65C & \\
\hline Bulgaria & 0 & 23.0 & & {$[37]$} \\
Croatia & 3.3 & 14.5 & 1.8 & {$[28]$} \\
Denmark & 5.6 & 12.8 & 1.8 & {$[38]$} \\
Ireland, north & 14 & 17.9 & & {$[4]$} \\
Italy, north & 3.2 & 13.4 & 1.3 & {$[29]$} \\
Italy, south & 1.5 & 14.0 & 0.5 & {$[30]$} \\
Finland & 4.6 & 9.8 & 2.3 & {$[31]$} \\
France & 7.7 & 14.0 & 1.95 & {$[18]$} \\
Portugal & 2.8 & 23.0 & & {$[32]$} \\
Russia & 3.7 & 13.3 & 1.7 & {$[33]$} \\
Slovenia & 4.0 & 14.5 & 0.5 & {$[28]$} \\
Spain, north & 3.0 & 20.0 & 1.0 & {$[34]$} \\
Spain, central & 2.0 & 16.0 & & {$[36]$} \\
Sweden & 6.2 & 11.4 & 1.6 & {$[35]$} \\
Sweden, Saami & 2.0 & 7.9 & 3.0 & {$[31]$} \\
Jordan & $\mathbf{0}$ & $\mathbf{1 1 . 2 5}$ & $\mathbf{0 . 1 1}$ & Present study \\
Saudi Arabia & 0 & $8.5-17.7$ & & {$[5,16]$} \\
Tunisia & 0.5 & 17.5 & & {$[17]$} \\
USA, Caucasian & 6.8 & 15.2 & 1.6 & {$[11]$} \\
USA, Hispanic & 2.7 & 12.4 & 0.6 & {$[11]$} \\
USA, Asian & 0.2 & 3.3 & 0 & {$[11]$} \\
USA, African & 1.1 & 5.1 & 1.7 & {$[11]$} \\
Ecuador & 0 & 3.5 & 4.0 & {$[12]$} \\
\hline
\end{tabular}

jority of the crusaders were of southern European origin having a low frequency of the C282Y. Searching cases for hemochromatosis in the largest tertiary healthcare hospital in northern Jordan which serves 1.5 million inhabitants we found only one case of hemochromatosis. Testing the patient revealed a single H63D mutant allele.

Our results were compared to data reported from different populations. As shown in Table 2, the prevalence of $\mathrm{C} 282 \mathrm{Y}$ variants is higher in North of Europe where the highest frequency is observed in areas between Nordic and Anglo-Saxon countries with rates fluctuating between $6 \%$ in Northern countries and $14 \%$ as reported for Ireland. In contrast, South of Europe, and in the Mediterranean countries the prevalence is very low. In countries such as Italy and France, highest frequency is located in the north $(3.2 \%, 7.7 \%$ respectively) compared with the south $(1.5 \%, 3.3 \%$, the later value not shown in Table 2). In our study, C282Y frequency $(0 \%)$ is less than those reported in Europe and USA but similar to those reported for countries of the Middle East and Africa (Table 2).

The S65C variant has not been investigated much and there is little information about its genetic frequency. There were no S65C homozygotes in our study and heterozygotes frequency was $0.23 \%$ of our population with an allele frequency of $0.11 \%$. The lowest allele frequency was reported in Asian Americans [11] and the highest was reported in Ecuador [12]. S65C, when combined with $\mathrm{C} 282 \mathrm{Y}$, is associated with an increased risk of hemochromatosis diagnosis [13]. Recently, S65C variant was found to have a lowering effect on iron status markers [14].

$\mathrm{H} 63 \mathrm{D}$ variant is more common than $\mathrm{C} 282 \mathrm{Y}$ and the geographical distribution of H63D variant in Europe is the reverse of that observed for $\mathrm{C} 282 \mathrm{Y}$ variant, following a decreasing line from south to north of Europe [15]. Most European populations being studied have an allele frequency of 10-20\%. Frequency above $20 \%$ were observed in Spanish, Portuguese and Bulgarians with the highest frequency being reported for Basque population $(30.4 \%)$ [5]. Our results show the H63D allele frequency to be $11.25 \%$ which seems higher than that found in Saudi Arabian population (8.5\%) [5], on the other hand, a recent report in the Saudi population estimated allele frequency to be as high as $17.7 \%$ [16]. The $11.25 \%$ allele frequency in Jordan is closer to that reported from European populations like UK, Sweden, Norway and Italy [5]. In addition, Jordanian H63D allele frequency is much less than that reported in Spain where the highest European frequency is found and is slightly less than that reported for Turkey, Greece, and North Africa [5,17]. No compound heterozygotes were found. Thus our results agree with the fact that H63D variant is not restricted to the European population. Although this variant is associated with the mild form of hemochromatosis, it shows incomplete penetrance which decreases the clinical impact of this variant [18].

We attempted to test the association of the three $H F E$ variants with diabetes, however, C282Y was completely absent from our study population and S65C was present in only one allele of the 880 alleles tested. H63D on the other hand was more frequent and allowed us to study its association with diabetes in the Jordanian Arab population. We did not find an association between H63D variant and diabetes in our cohort. This is consistent with a recent data from polish [19], Greek [20], British [21], American [22], Iranian [23], Australian [24], and Irish [25] populations. In contrast, one study in the Polish population found an increase in H63D allele carrier frequency in patients with diabetic nephropathy [26]. In a study in Spain, authors found increased frequency of H63D allele in patients of type 2 diabetes, however, they had marginal significance with a $\mathrm{p}$ value of 0.04 , and a low number of control cases, 108 [27].

Diabetes is the most commonly observed endocrine disorder in people with hemochromatosis which is due 
Table 3

Genotype and allele frequencies of H63D in tested individuals with Diabetes Mellitus and blood donors (controls)

\begin{tabular}{lrcrrrr}
\hline & \multicolumn{2}{c}{ Diabetics } & & \multicolumn{2}{c}{ Blood donors } & \\
\cline { 2 - 3 } H63D $($ c.187C $>$ G) & $\mathrm{N}$ & $\%$ & & $\mathrm{~N}$ & $\%$ & \\
\hline GG genotype & 0 & 0 & & 0 & 0 & 0.84 \\
CG genotype & 21 & 23.60 & & 46 & 22.55 & \\
CC genotype & 68 & 76.40 & & 158 & 77.45 & \\
G allele & 21 & 11.80 & & 46 & 11.27 & 0.65 \\
C allele & 157 & 88.20 & & 362 & 88.73 & \\
\hline
\end{tabular}

to accumulation of iron which alters glucose and insulin homeostasis as seen in type 2 diabetes even if it is present in a lesser extent as observed in $H F E$ heterozygotes. Since there was a repeated suggestion of an association between the inheritance of $H F E$ variants and diabetes mellitus, and due to the high prevalence of diabetes in Jordan, it was rational to investigate if there is a relationship between $\mathrm{H} 63 \mathrm{D}$ variant and having diabetes. However, there was no genetic difference between the control and the test group which suggests that H63D variant does not play a significant role in the development of diabetes mellitus in the Jordanian population and that other genetic and environmental factors may be considered. One limitation to mention is the lower age average in the controls as compared to cases. We re-analyzed the data considering half the controls, those with the higher age (102 control subjects, average age 33.0 years) and we still did not detect a significant association (data not shown).

In conclusion, we analyzed the frequency of the three hemochromatosis HFE variants; C282Y, H63D, and S65C in Jordanian Arab population. C282Y, the major disease causing mutation was completely absent from our 440 individuals tested. This is consistent with the fact that hemochromatosis has a very low prevalence in Jordan and that it is not merely under-diagnosed. S65C variant was found in a single allele. H63D variant was found to be common with $11.25 \%$ allele frequency, but it plays a minor role in hemochromatosis when compared to C282Y mutation. Furthermore, we analyzed the association of H63D variant with the risk of developing diabetes but we could not detect significant association in our test group suggesting that this variant does not play a major role in increasing the risk of developing diabetes in Jordanian Arab population.

\section{Acknowledgments}

We would like to thank all participants in this study. We thank Dr. Osama Smadi and Dr. Richard Allen for providing us with positive controls. This work was supported by a grant from the Jordan University of Science and Technology \#59/2008.

\section{References}

[1] P.C. Adams and J.C. Barton, Haemochromatosis, Lancet 370 (2007), 1855-1860.

[2] J.N. Feder, A. Gnirke, W. Thomas et al., A novel MHC class I-like gene is mutated in patients with hereditary haemochromatosis, Nat Genet 13 (1996), 399-408.

[3] A. Pietrangelo, Hereditary hemochromatosis- A new look at an old disease, New Eng J Med 350 (2004), 2383-2397.

[4] E. Ryan, C. O'keane and J. Growe, Hemochromatosis in Ireland and HFE, Blood Cells Mol Dis 31 (1998), 428-432.

[5] A.T. Merryweather-Clarke, J.J. Pointon, J.D. Shearman and K.J. Robson, Global prevalence of putative haemochromatosis mutations, J Med Genet 34 (1997), 275-278.

[6] A. Asberg, K. Hveem, K. Kannelonning and W.O. Irgens, Penetrance of the C28Y/C282Y genotype of the HFE gene, Scand J Gastroenterol 42 (2007), 1073-1077.

[7] K.B. Mullis and F.A. Faloona, Specific synthesis of DNA in vitro via a polymerase-catalyzed chain reaction, Methods Enymol 155 (1987), 335-350.

[8] S. Rozen and H. Skaletsky, Primer3 on the WWW for general users and for biologist programmers, Methods Mol Biol 132 (2000), 365-386.

[9] G.H. Freeman and J.H. Halton, Note on an exact treatment of contingency, goodness of fit and other problems of significance, Biometrika 38 (1951), 141-149.

[10] N. Milman and P. Pedersen, Evidence that the Cys282Tyr mutation of the HFE gene originated from a population in Southern Scandanavia and spread with the Vikings, Clin Genet 64 (2003), 36-47.

[11] E. Beutler, V. Felitti and T. Gelbart, The effect of HFE genotypes on measurements of iron overload in patients attending a health appraisal clinic, Ann Intern Med 133 (2000), 329-337.

[12] P.E. Leone, P. Gimenez, J.C. Collantes and C.P. Mino, Analysis of HFE gene mutations (C282Y, H63D, and S65C) in the Ecuadorian population, Ann Hematol 84 (2005), 103-106.

[13] A. Aesberg, K. Thorstensen, K. Hveem and K.S. Bjerve, Hereditary hemochromatosis: the clinical significance of the S65C mutation, Genet Test 6 (2002), 59-62.

[14] P. Pedersen and N. Milman, Genetic screening for HFE hemochromatosis in 6,020 Danish men: penetrance of C282Y, H63D, and S65C variants, Ann Hematol DOI 10.1007/s00277008-06791.

[15] E. Hanson, G. Imperatore and W. Burke, HFE gene and hereditary hemochromatosis a HuGE review, Am J of Epidemiol 154 (2001), 193-206. 
[16] O.A. Alsmadi, F. Al-Kayal, M. Al-Hamed and B.F. Meyer, Frequency of common HFE variants in the Saudi population: a high throughput molecular beacon-based study, BMC Med Genet 7 (2006), 43-49.

[17] R. Sassi, S. Hmida, H. Kaabi et al., Prevalence of C282Y and H63D mutations in the hemochromatosis (HFE) gene in Tunisian population, Ann Genet 47 (2004), 325-330.

[18] C. Mura, O. Raguenes and C. Ferec, HFE mutaions analysis in 711 hemochromatosis probands: Evidence for S65C implication in mild forms of hemochromatosis, Blood 93 (1999), 2502-2505.

[19] M.T. Malecki, T. Klupa, M. Walus et al., A search for association between hereditary hemochromatosis HFE gene mutations and type 2 diabetes mellitus in a polish population, Med Sci Monit 9 (2003), BR91-95.

[20] I.G. Habeos, A. Psyrogiannis, V. Kyriazopoulou et al., The role of Hemochromatosis $\mathrm{C} 282 \mathrm{Y}$ and H63D mutations in the development of type 2 diabetes millitus in Greece, Hormones 2 (2003), 55-60.

[21] D.J. Halsall, I. McFarlane, J. Luan et al., Typical type 2 diabetes mellitus and HFE gene mutations: a population-based case-control study, Hum Mol Genet 12 (2003), 1361-1365.

[22] R.T. Acton, J.C. Barton, L.V. Passmore et al., Relationships of serum ferritin, transferring saturation, and $H F E$ mutations and self-reported diabetes in the hemochromatosis and iron overload screening (HEIRS) study, Diabetes Care 29 (2006), 2084-2089.

[23] F. Sharifi, A. Esmaeilzadeh and M. Zali, Hemochromatosis gene (HFE) mutations in patients with type 2 diabetes and their control group in an Iranian population, Saudi Med J 29 (2008), 808-812.

[24] T.M.E. Davis, J. Beilby, W.A. Davis et al., Prevalence, characteristics, and prognostic significance of $H F E$ gene mutations in Type 2 diabetes, Diabetes Care 31 (2008), 1795-1801.

[25] L. Kirk, J. Bird, S. Ramadan et al., Hemochromatosis gene frequency in a control and diabetic Irish population, Ir J Med Sci 178 (2008), 39-42.

[26] D.K. Moczulski, W. Grzeszczak and B. Gawlik, Role of hemochromatosis C282Y and H63D mutations in HFE gene in development of type 2 diabetes and diabetic nephropathy, Diabetes Care 24 (2001), 1187-1191.
27] J.-M. Fernandez-Real, J. Vendrell, M. Baiget et al., C282Y and H63D mutations of the hemochromatosis candidate gene in type 2 diabetes, Diabetes Care 22 (1999), 525-526.

[28] S. Ristic, J. Makuc, N. Starcevic et al., Hemochromatosis gene mutations in the Croatian and Slovanian population, Clin Genet 64 (2003), 444-446.

[29] R. Mariani, A. Slavioni, C. Corengia et al., Prevalence of HFE mutaions in upper Northern Italy: Study of 1132 unrelated blood donors, Dig Liver Dis 35 (2003), 479-481.

[30] A. Pietrapertosa, A. Vitucci, D. Campanale et al., HFE gene mutations in Apulin population: allele frequencies, Eur $J$ Epidemiol 18 (2003), 685-689.

[31] L.E. Beckman, K. Sjoberg, S. Eriksson and L. Beckman, Hemochromatosis gene mutations in Finns, Sweedes and Swedish Saamis, Hum Hered 52 (2001), 110-112.

[32] G. Porto, H. Alves, P. Rodrigues et al., Major histocompatability complex class I associations in iron overload: evidence for a new link between the HFE H63D mutation, HLAA29, and non-classical forms of hemochromatosis, Immunogenetics $\mathbf{4 7}$ (1998), 404-410.

[33] S.V. Mikhalova, V.F. Kobazev, I.V. Kulikov et al., Polymorphism of the HFE gene associated with hereditary hemochromatosis in populations of Russia, Genetika 39 (2003), 988995.

[34] A. Altes, A. Ruiz, M.J. Barcelo et al., Prevalence of the C282Y, H63D, and S65C mutations of the HFE gene in 1,146 newborns from a region of northern Spain, Genet Test 8 (2004), 407-410.

[35] P. Holmstrom, J. Marmur, G. Eggersten et al., Mild iron overload in patients carrying the HFE S65C gene mutation: a retrospective study in patients with suspected iron overload and healthy controls, Gut 51 (2002), 723-730.

[36] S. Alvarez, M.S. Mesa, F. Bandres and E. Arroyo, C282Y and H63D mutation frequencies in a population from central Spain, Dis Markers 17 (2001),111-114.

[37] A. Ivanova, N. von Ahsen, D. Adjarov et al., C282Y and H63D mutations in the HFE gene are not associated with porphyria cutanea tarda in Bulgaria, Hepatology 30 (1999), 1531-1532.

[38] P. Pederson, G.V. Melson and N. Milman, Frequencies of the haemochromatosis gene (HFE) variants C282Y, H63D and S65C in 6,020 ethnic Danish men, Ann Hematol 87 (2008), $735-740$. 


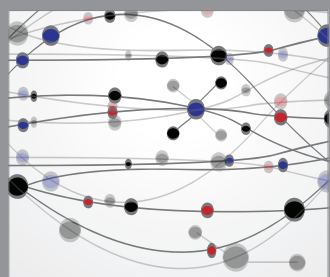

The Scientific World Journal
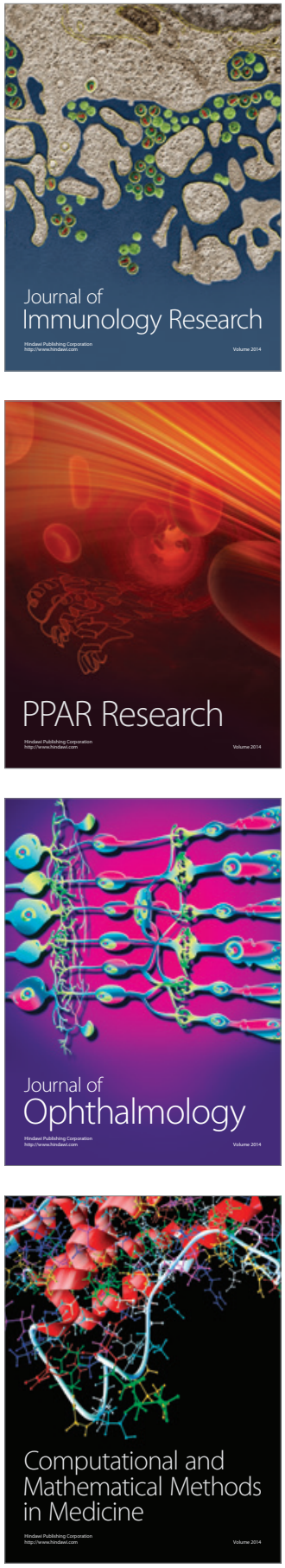

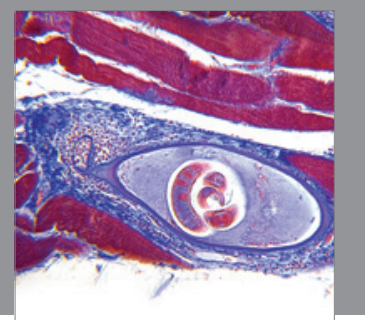

Gastroenterology

Research and Practice
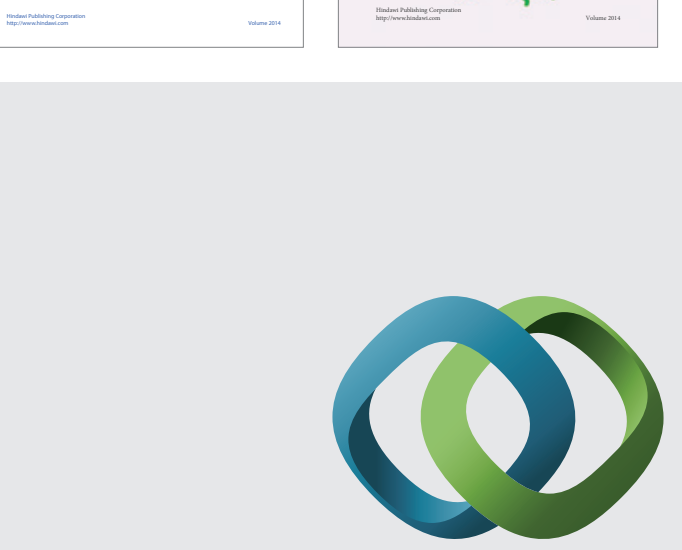

\section{Hindawi}

Submit your manuscripts at

http://www.hindawi.com
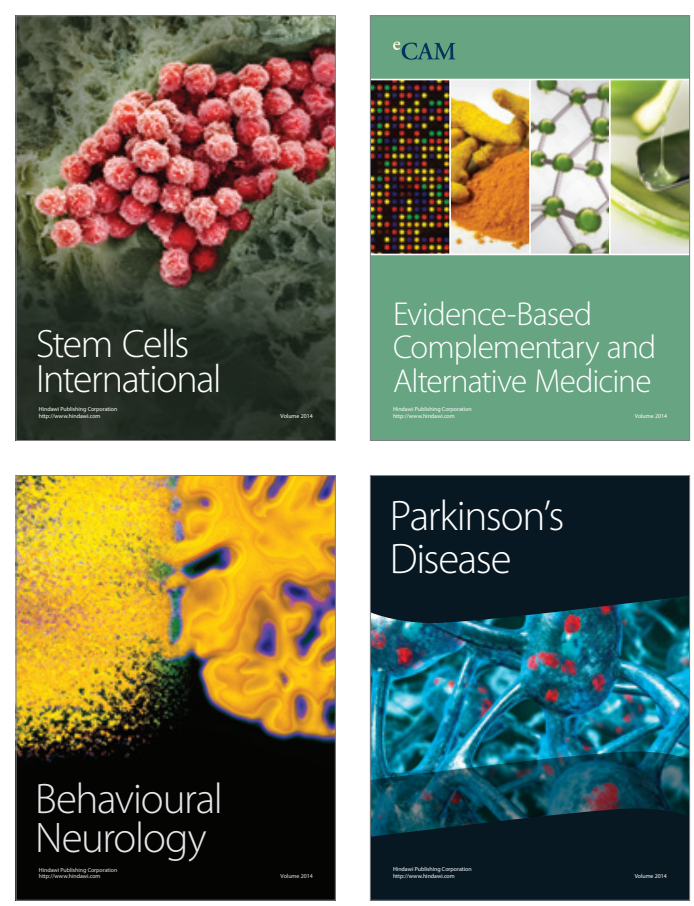

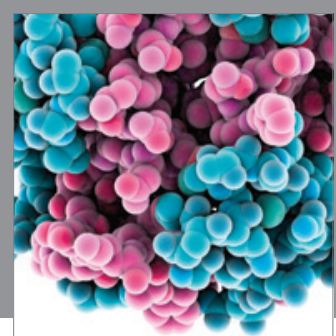

Journal of
Diabetes Research

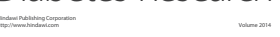

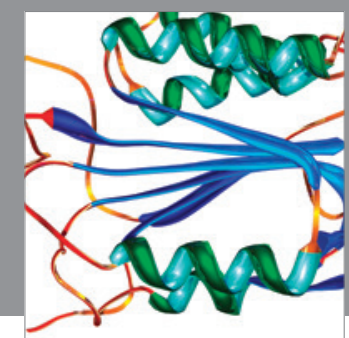

Disease Markers
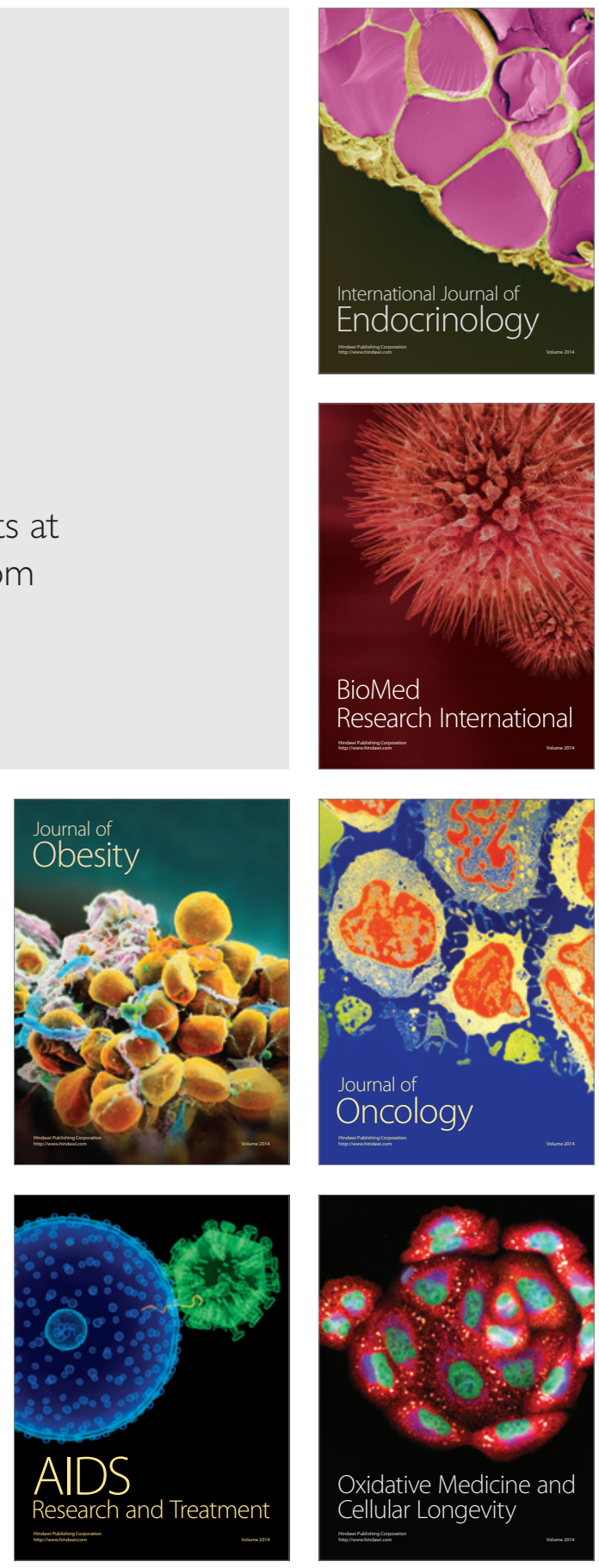